\title{
Convenient One-Pot Synthesis of Multisubstituted Tetrahydropyrimidines via Catalyst-Free Multicomponent Reactions
}

Min Zhang, Huanfeng Jiang*, Hailing Liu, and Qiuhua Zhu

The College of Chemistry, South China University of Technology, Guangzhou 510640, P. R.

China

General procedure: All reactions were performed at the desired temperature under air atmosphere in a round bottom flask equipped with magnetic stir bar. ${ }^{1} \mathrm{H}$ NMR spectra were recorded using a Bruker Avance $400 \mathrm{MHz}$ NMR spectrometer and referenced to $7.24 \mathrm{ppm}$ for chloroform solvent with TMS as internal standard. IR spectra were obtained as potassium bromide pellets or as liquid films between two potassium bromide pellets with a Brucker Vector 22 spectrometer. TLC was performed using commercially prepared 100-400 mesh silica gel plates (GF254), and visualization was effected at $254 \mathrm{~nm}$. All the other chemicals were purchased from Aldrich Chemicals.

General procedure for one-pot synthesis of tetrasubstituted tetrahydropyrimidines via three-component reactions (A): To a stirring mixture of but-2-ynedioic acid diethyl ester (170mg, $1 \mathrm{mmol}$ ) and aniline (196mg, $2.1 \mathrm{mmol}), 2 \mathrm{~mL}$ DMF and $35 \%$ formaldehyde $(4 \mathrm{mmol}$, $345 \mathrm{mg}$ ) were added successively. The mixture was stirred at $100{ }^{\circ} \mathrm{C}$ for $3 \mathrm{~h}$. After completion of the reaction (monitored by TLC), the mixture was cooled to room temperature, diluted with water and extracted with diethyl ether $(15 \mathrm{~mL} \times 5)$. The ether layer was washed with saturated 
brine $(10 \mathrm{~mL} \times 5)$, and dried with anhydrous $\mathrm{MgSO}_{4}$. The solvent was removed in vacuo and the crude product was purified by preparative TLC with hexane / ethyl acetate (10:1) as the eluent to afford the desired product $4 \mathbf{a a}(338 \mathrm{mg}, 89 \%)$ as a yellowish oil.

General procedure for one-pot synthesis of tetrasubstituted tetrahydropyrimidines via four-component reactions (B): To a stirring mixture of but-2-ynedioic acid diethyl ester (170mg, 1mmol) aniline (93mg, $1 \mathrm{mmol}), 2 \mathrm{~mL}$ DMF, benzylamine (118mg, $1.1 \mathrm{mmol}), 35 \%$ formaldehyde ( $4 \mathrm{mmol}, 345 \mathrm{mg}$ ) were added successively. The mixture was stirred at $100^{\circ} \mathrm{C}$ for 1h. After completion of the reaction (monitored by TLC), the mixture was cooled to room temperature, diluted with water and extracted with diethyl ether $(15 \mathrm{~mL} \times 5)$, and then the ether layer was washed with saturated brine $(10 \mathrm{~mL} \times 5)$, dried with anhydrous $\mathrm{MgSO}_{4}$. After the solvent was removed in vacuo, the crude product was purified by preparative TLC with hexane / ethyl acetate (10:1) as the eluent to afford the desired product 4ab (366mg, 93\%) as a yellowish oil.

The following compounds in Table 2 and Scheme 1 were prepared according to General procedure A.

(1) 1, 3-diphenyl- 1, 2, 3, 6-tetrahydropyrimidine-4, 5-dicarboxylic acid diethyl ester (4aa):

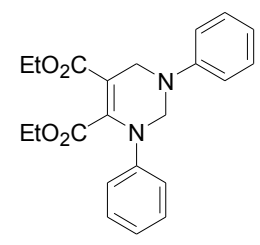

Yellowish oil; IR $(\mathrm{KBr})$ : $\mathrm{V}_{\max }=2982,1740,1697,1598,1498,1397,1274,1164,1109$, $1041 \mathrm{~cm}^{-1} ;{ }^{1} \mathrm{H}$ NMR $\left(400 \mathrm{MHz}, \mathrm{CDCl}_{3}\right): \delta=7.25-6.87(\mathrm{~m}, 10 \mathrm{H}), 4.90(\mathrm{~s}, 2 \mathrm{H}), 4.25(\mathrm{~s}, 2 \mathrm{H}), 4.20$ (q, J=7.2Hz, 2H), 4.00 (q, J=7.2Hz, 2H), 1.26(t, J=7.2Hz, 3H), 0.97ppm(t, J=7.2Hz, 3H); MS 
(EI): $\mathrm{m} / \mathrm{z}(\%): 380\left(\mathrm{M}^{+}, 24\right), 202(100)$; Anal. Calcd for $\mathrm{C}_{22} \mathrm{H}_{24} \mathrm{~N}_{2} \mathrm{O}_{4}$ : C, 69.46; $\mathrm{H}, 6.36 ; \mathrm{N}, 7.36$; Found: C, 69.18; H, 6.54; N, 6.99 .

(2) 1, 3-dibenzyl- 1, 2, 3, 6-tetrahydropyrimidine-4, 5-dicarboxylic acid diethyl ester (4bb):

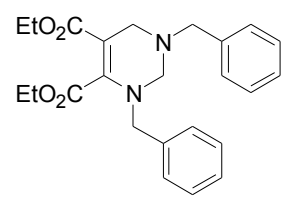

Yellowish oil; IR (KBr): $v_{\max }=3448,2980,1737,1686,1581,1496,1395,1285,1260,1079$, $1042 \mathrm{~cm}^{-1} ;{ }^{1} \mathrm{H}$ NMR $\left(400 \mathrm{MHz}, \mathrm{CDCl}_{3}\right): \delta=7.29-7.14(\mathrm{~m}, 10 \mathrm{H}), 4.35$ (q, J=7.2Hz, 2H), 4.16(s, $2 \mathrm{H}), 4.11(\mathrm{q}, \mathrm{J}=7.2 \mathrm{~Hz}, 2 \mathrm{H}), 3.81(\mathrm{~s}, 2 \mathrm{H}), 3.59(\mathrm{~s}, 2 \mathrm{H}), 3.55(\mathrm{~s}, 2 \mathrm{H}), 1.30(\mathrm{t}, \mathrm{J}=7.2 \mathrm{~Hz}, 3 \mathrm{H})$, 1.21ppm(t, J=7.2Hz, 3H); MS (El): m/z (\%): 408(M+, 12); Anal. Calcd for $\mathrm{C}_{24} \mathrm{H}_{28} \mathrm{~N}_{2} \mathrm{O}_{4}: \mathrm{C}, 70.37$; H, 6.91; N, 6.86; Found: C, 70.51; H, 7.08; N, 6.26.

(3) 1, 3-dibutyl- 1, 2, 3, 6-tetrahydropyrimidine-4, 5-dicarboxylic acid diethyl ester (4cc):

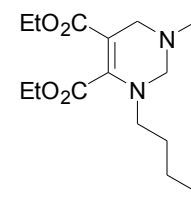

Yellowish oil; IR $(\mathrm{KBr}): \mathrm{v}_{\max }=3446,2960,1740,1685,1581,1460,1399,1283,1245,1145$, $1039 \mathrm{~cm}^{-1} ;{ }^{1} \mathrm{H}$ NMR $\left(400 \mathrm{MHz}, \mathrm{CDCl}_{3}\right): \delta=4.33(\mathrm{q}, \mathrm{J}=7.2 \mathrm{~Hz}, 2 \mathrm{H}), 4.07$ (q, J=7.2Hz, 2H), 3.93(s, 2H), $3.46(\mathrm{~s}, 2 \mathrm{H}), 2.99(\mathrm{t}, \mathrm{J}=7.6 \mathrm{~Hz}, 2 \mathrm{H}), 2.49(\mathrm{t}, \mathrm{J}=7.6 \mathrm{~Hz}, 2 \mathrm{H}), 1.50-1.35(\mathrm{~m}, 4 \mathrm{H}), 1.34-1.32(\mathrm{~m}$, 4H), 1.26-1.17 (m, 6H); 0.92-0.87ppm (m, 6H); MS (El): m/z (\%): 340(M+, 22); Anal. Calcd for $\mathrm{C}_{18} \mathrm{H}_{32} \mathrm{~N}_{2} \mathrm{O}_{4}: \mathrm{C}, 63.50 ; \mathrm{H}, 9.47 ; \mathrm{N}, 8.23$; Found: $\mathrm{C}, 63.71 ; \mathrm{H}, 9.55 ; \mathrm{N}, 7.92$.

(4) 1, 3-di-p-tolyl- 1, 2, 3, 6-tetrahydropyrimidine-4, 5-dicarboxylic acid diethyl ester (4dd): 
<smiles>CCOC(=O)C1=C(C(=O)OCC)N(c2ccc(C)cc2)CN(c2ccc(C)cc2)C1</smiles>

Yellowish oil; IR (KBr): $v_{\max }=3459,2982,1740,1692,1591,1514,1395,1261,1162,1109$, $1039 \mathrm{~cm}^{-1} ;{ }^{1} \mathrm{H}$ NMR $\left(400 \mathrm{MHz}, \mathrm{CDCl}_{3}\right): \delta=7.05-6.81(\mathrm{~m}, 8 \mathrm{H}), 4.82(\mathrm{~s}, 2 \mathrm{H}), 4.20(\mathrm{~s}, 2 \mathrm{H}), 4.16(\mathrm{q}$, $\mathrm{J}=7.2 \mathrm{~Hz}, 2 \mathrm{H}), 4.00(\mathrm{q}, \mathrm{J}=7.2 \mathrm{~Hz}, 2 \mathrm{H}), 2.28(\mathrm{~s}, 3 \mathrm{H}), 2.24(\mathrm{~s}, 3 \mathrm{H}), 1.24(\mathrm{t}, \mathrm{J}=7.2 \mathrm{~Hz}, 3 \mathrm{H})$, 0.99ppm(t, J=7.2Hz, 3H); MS (El): m/z (\%): 408(M+22), 216(100); Anal. Calcd for $\mathrm{C}_{24} \mathrm{H}_{28} \mathrm{~N}_{2} \mathrm{O}_{4}$ : C, 70.57; H, 6.91; N, 6.86; Found: C, 70.28; H, 7.12; N, 6.50.

(5) 1,3-bis-(4-fluo-phenyl)-1,2,3,6-tetrahydropyrimidine-4,5-dicarboxylic acid diethyl ester (4ee):

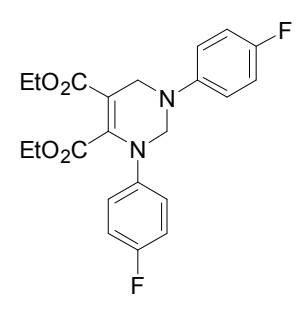

Yellowish solid; IR (KBr): $v_{\max }=3455,2985,1739,1693,1590,1464,1374,1269,1224,1159$, 1109, $1038 \mathrm{~cm}^{-1} ;{ }^{1} \mathrm{H}$ NMR $\left(400 \mathrm{MHz}, \mathrm{CDCl}_{3}\right): \delta=6.93-6.87(\mathrm{~m}, 8 \mathrm{H}), 4.77(\mathrm{~s}, 2 \mathrm{H}), 4.18(\mathrm{~s}, 2 \mathrm{H})$, $4.17(\mathrm{q}, \mathrm{J}=7.2 \mathrm{~Hz}, 2 \mathrm{H}), 3.99(\mathrm{q}, \mathrm{J}=7.2 \mathrm{~Hz}, 2 \mathrm{H}), 1.25(\mathrm{t}, \mathrm{J}=7.2 \mathrm{~Hz}, 3 \mathrm{H}), 0.98 \mathrm{ppm}(\mathrm{t}, \mathrm{J}=7.2 \mathrm{~Hz}, 3 \mathrm{H})$; MS (El): $\mathrm{m} / \mathrm{z}(\%): 416\left(\mathrm{M}^{+}, 9\right), 220(100) ;$ Anal. Calcd for $\mathrm{C}_{22} \mathrm{H}_{22} \mathrm{~F}_{2} \mathrm{~N}_{2} \mathrm{O}_{4}: \mathrm{C}, 63.45 ; \mathrm{H}, 5.33 ; \mathrm{N}$, 6.73; Found: C, 63.58; H, 6.92; N, 6.38.

The following compounds in Table 2 and Scheme 1 were prepared according to General procedure $B$.

(6) 1-benzyl-3-phenyl- 1, 2, 3, 6-tetrahydropyrimidine-4, 5-dicarboxylic acid diethyl ester (4ab): 


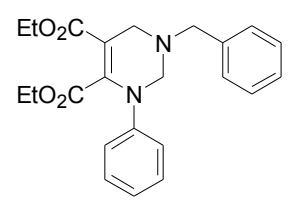

Yellowish solid; IR (KBr): $v_{\max }=3447,2981,1739,1680,1582,1458,1394,1260,1208,1109$, $1038 \mathrm{~cm}^{-1} ;{ }^{1} \mathrm{H}$ NMR $\left(400 \mathrm{MHz}, \mathrm{CDCl}_{3}\right): \delta=7.30-7.11(\mathrm{~m}, 10 \mathrm{H}), 4.33(\mathrm{~s}, 2 \mathrm{H}), 4.13(\mathrm{q}, \mathrm{J}=7.2 \mathrm{~Hz}$, 2H), 4.05(q, J=7.2Hz, 2H), 3.80(s, 2H), 3.70 (s $2 \mathrm{H}), 1.21$ (t, J=7.2Hz, 3H), $1.01 \mathrm{ppm}(\mathrm{t}, \mathrm{J}=7.2 \mathrm{~Hz}$,

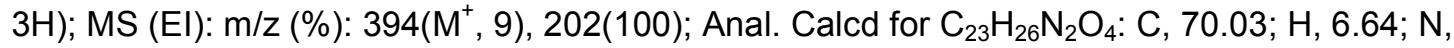
7.10; Found: C, 69.68; H, 6.84; N, 6.66 .

(7) 3-benzyl-1-phenyl- 1, 2, 3, 6-tetrahydropyrimidine-4, 5-dicarboxylic acid diethyl ester (4ba):

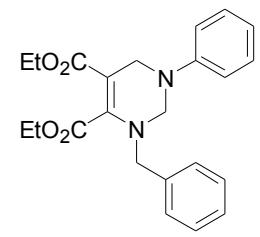

Yellowish oil; IR (KBr): $v_{\max }=3446,2984,1742,1681,1579,1456,1392,1269,1208,1106$, $1038 \mathrm{~cm}^{-1} ;{ }^{1} \mathrm{H}$ NMR $\left(400 \mathrm{MHz}, \mathrm{CDCl}_{3}\right): \delta=7.28-7.16(\mathrm{~m}, 7 \mathrm{H}), 6.80-6.78(3 \mathrm{H}), 4.39(\mathrm{~s}, 2 \mathrm{H}), 4.29$ (q, J=7.2Hz, 2H), 4.21(s, 2H), 4.17 (q, J=7.2Hz, 2H), 4.09(s, 2H), 1.30-1.19ppm(m, 6H); MS (EI): m/z (\%): 394(M $\left.\mathrm{M}^{+}, 25\right), 303(33)$; Anal. Calcd for $\mathrm{C}_{23} \mathrm{H}_{26} \mathrm{~N}_{2} \mathrm{O}_{4}$ : C, 70.03; $\mathrm{H}, 6.64 ; \mathrm{N}, 7.10$.

(8) 1-butyl-3-phenyl- 1, 2, 3, 6-tetrahydropyrimidine-4, 5-dicarboxylic acid diethyl ester (4ac):

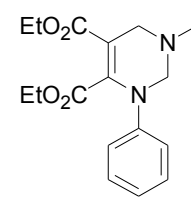

Yellowish oil; IR (KBr): $v_{\max }=3448,2960,1740,1693,1581,1496,1461,1399,1250,1211$, 1172, 1105, $1056 \mathrm{~cm}^{-1} ;{ }^{1} \mathrm{H}$ NMR $\left(400 \mathrm{MHz}, \mathrm{CDCl}_{3}\right): \delta=7.30-7.10(\mathrm{~m}, 5 \mathrm{H}), 4.36(\mathrm{~s}, 2 \mathrm{H}), 4.13(\mathrm{q}$, $\mathrm{J}=7.2 \mathrm{~Hz}, 2 \mathrm{H}), 4.01(\mathrm{q}, \mathrm{J}=7.2 \mathrm{~Hz}, 2 \mathrm{H}), 3.64(\mathrm{~s}, 2 \mathrm{H}), 2.61(\mathrm{t}, \mathrm{J}=7.2 \mathrm{~Hz}, 2 \mathrm{H}), 1.43-1.39(\mathrm{~m}, 2 \mathrm{H})$, 
1.32-1.26 (m, 2H), $1.22(\mathrm{t}, \mathrm{J}=7.2 \mathrm{~Hz}, 3 \mathrm{H}), 0.98(\mathrm{t}, \mathrm{J}=7.2 \mathrm{~Hz}, 3 \mathrm{H}), 0.85 \mathrm{ppm}(\mathrm{t}, \mathrm{J}=7.2 \mathrm{~Hz}, 3 \mathrm{H}) ; \mathrm{MS}$ (El): m/z (\%): 360(M+, 11), 202(100); Anal. Calcd for $\mathrm{C}_{20} \mathrm{H}_{28} \mathrm{~N}_{2} \mathrm{O}_{4}: \mathrm{C}, 66.64 ; \mathrm{H}, 7.83 ; \mathrm{N}, 7.77$; Found: C, 66.38; H, 8.15; N, 7.64.

(9)3-phenyl-1-p-tolyl- 1, 2, 3, 6-tetrahydropyrimidine-4, 5-dicarboxylic acid diethyl ester (4ad):

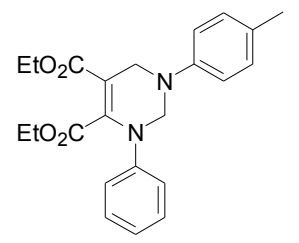

Yellowish solid; IR (KBr): $v_{\max }=3447,2981,1740,1680,1582,1514,1457,1394,1260,1208$, 1109, $1038 \mathrm{~cm}^{-1} ;{ }^{1} \mathrm{H}$ NMR $\left(400 \mathrm{MHz}, \mathrm{CDCl}_{3}\right): \delta=7.26-6.80(\mathrm{~m}, 9 \mathrm{H}), 4.85(\mathrm{~s}, 2 \mathrm{H}), 4.21(\mathrm{~s}, 2 \mathrm{H})$, 4.19(q, J=7.2Hz, 2H), 4.00(q, J=7.2Hz, 2H), $2.23(\mathrm{~s}, 3 \mathrm{H}), 1.25$ (t, J=7.2Hz, 3H), 0.97ppm(t, $\mathrm{J}=7.2 \mathrm{~Hz}, 3 \mathrm{H}$ ); $\mathrm{MS}(\mathrm{EI}): \mathrm{m} / \mathrm{z}(\%): 394\left(\mathrm{M}^{+}, 16\right), 202(100)$; Anal. Calcd for $\mathrm{C}_{23} \mathrm{H}_{26} \mathrm{~N}_{2} \mathrm{O}_{4}: \mathrm{C}, 70.03$; H, 6.64; N, 7.10; Found: C, 69.83; H, 6.90; N, 6.93

(10) 1-(4-fluoro-phenyl)-3-phenyl- 1, 2, 3, 6-tetrahydropyrimidine-4, 5-dicarboxylic acid diethyl ester (4ae):

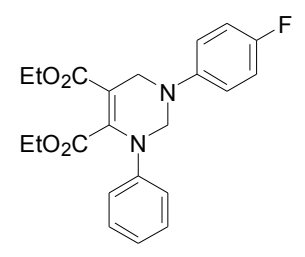

Yellowish oil; IR (KBr): $v_{\max }=3446,2983,1739,1692,1582,1458,1399,1259,1228,1158$, 1107, $1036 \mathrm{~cm}^{-1} ;{ }^{1} \mathrm{H}$ NMR (400 MHz, $\left.\mathrm{CDCl}_{3}\right): \delta=7.25-6.87(\mathrm{~m}, 9 \mathrm{H}), 4.83(\mathrm{~s}, 2 \mathrm{H}), 4.19(\mathrm{~s}, 2 \mathrm{H})$, $4.17(q, J=7.2 \mathrm{~Hz}, 2 \mathrm{H}), 4.00(q, J=7.2 \mathrm{~Hz}, 2 \mathrm{H}), 1.25(\mathrm{t}, \mathrm{J}=7.2 \mathrm{~Hz}, 3 \mathrm{H}), 0.96 p p m(\mathrm{t}, \mathrm{J}=7.2 \mathrm{~Hz}, 3 \mathrm{H})$; 
MS (El): m/z (\%): 398(M+1 12); Anal. Calcd for $\mathrm{C}_{22} \mathrm{H}_{23} \mathrm{FN}_{2} \mathrm{O}_{4}: \mathrm{C}, 66.32 ; \mathrm{H}, 5.824 ; \mathrm{N}, 7.03$; Found: C, $66.04 ; \mathrm{H}, 6.14 ; \mathrm{N}, 7.20$.

(11) 3-phenyl-1-o-tolyl- 1, 2, 3, 6-tetrahydropyrimidine-4, 5-dicarboxylic acid diethyl ester (4af):

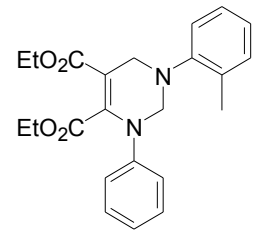

Yellowish oil; IR (KBr): $v_{\max }=3430,2981,1739,1694,1582,1495,1458,1394,1272,1211$, 1157, $1036 \mathrm{~cm}^{-1} ;{ }^{1} \mathrm{H}$ NMR $\left(400 \mathrm{MHz}, \mathrm{CDCl}_{3}\right): \delta=7.29-6.67(\mathrm{~m}, 9 \mathrm{H}), 4.87(\mathrm{~s}, 2 \mathrm{H}), 4.23(\mathrm{~s}, 2 \mathrm{H})$, 4.18(q, J=7.2Hz, 2H), 4.01(q, J=7.2Hz, 2H), $2.22(\mathrm{~s}, 3 \mathrm{H}), 1.26(\mathrm{t}, \mathrm{J}=7.2 \mathrm{~Hz}, 3 \mathrm{H}), 0.98 p p m(\mathrm{t}$, $\mathrm{J}=7.2 \mathrm{~Hz}, 3 \mathrm{H}$ ); $\mathrm{MS}(\mathrm{EI}): \mathrm{m} / \mathrm{z}(\%): 394\left(\mathrm{M}^{+}, 15\right), 202(100)$; Anal. Calcd for $\mathrm{C}_{23} \mathrm{H}_{26} \mathrm{~N}_{2} \mathrm{O}_{4}: \mathrm{C}, 70.03$; H, 6.64; N, 7.10; Found: C, 70.34; H, 7.02; N, 7.25.

(12) 3-phenyl-1-m-tolyl- 1, 2, 3, 6-tetrahydropyrimidine-4, 5-dicarboxylic acid diethyl ester (4ag):

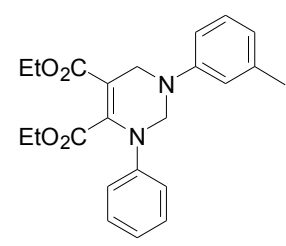

Yellowish oil; IR (KBr): $v_{\max }=3448,2981,1740,1693,1583,1495,1459,1398,1261,1232$, 1168, $1040 \mathrm{~cm}^{-1} ;{ }^{1} \mathrm{H}$ NMR $\left(400 \mathrm{MHz}, \mathrm{CDCl}_{3}\right): \delta=7.24-6.96(\mathrm{~m}, 9 \mathrm{H}), 4.61(\mathrm{~s}, 2 \mathrm{H}), 4.17(\mathrm{q}$, $\mathrm{J}=7.2 \mathrm{~Hz}, 2 \mathrm{H}), 4.07(\mathrm{~s}, 2 \mathrm{H}), 4.05(\mathrm{q}, \mathrm{J}=7.2 \mathrm{~Hz}, 2 \mathrm{H}), 2.20(\mathrm{~s}, 3 \mathrm{H}), 1.26(\mathrm{t}, \mathrm{J}=7.2 \mathrm{~Hz}, 3 \mathrm{H})$, 0.98ppm(t, J=7.2Hz, 3H); MS (EI): m/z (\%): 394(M+2 23), 202(100); Anal. Calcd for $\mathrm{C}_{23} \mathrm{H}_{26} \mathrm{~N}_{2} \mathrm{O}_{4}$ : C, 70.03; H, 6.64; N, 7.10; Found: C, 70.26; H, 7.03; N, 7.05. 


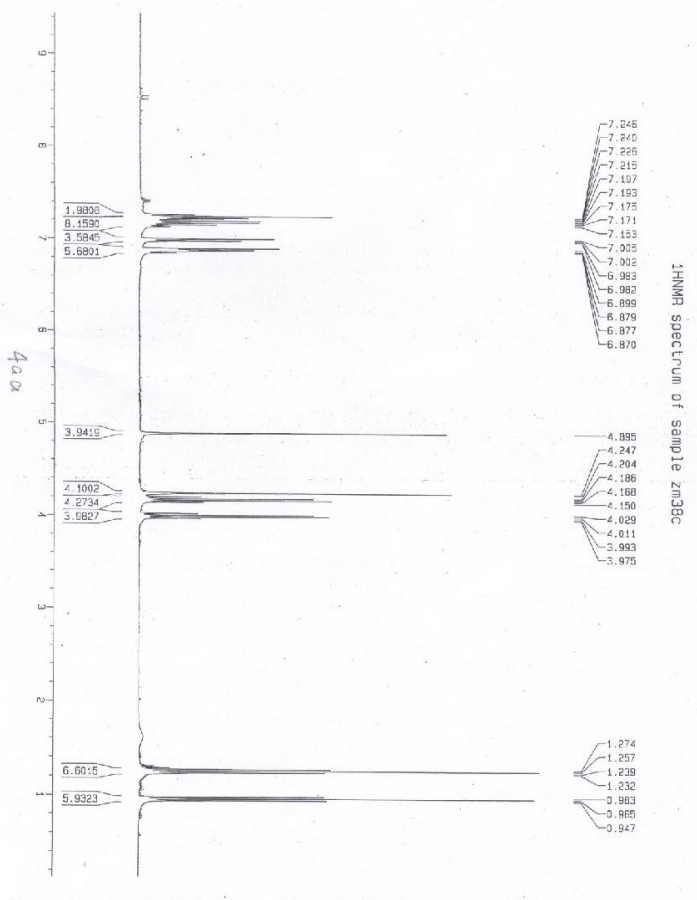

${ }^{1} \mathrm{H}$ NMR Spectrum of $4 \mathrm{aa}$

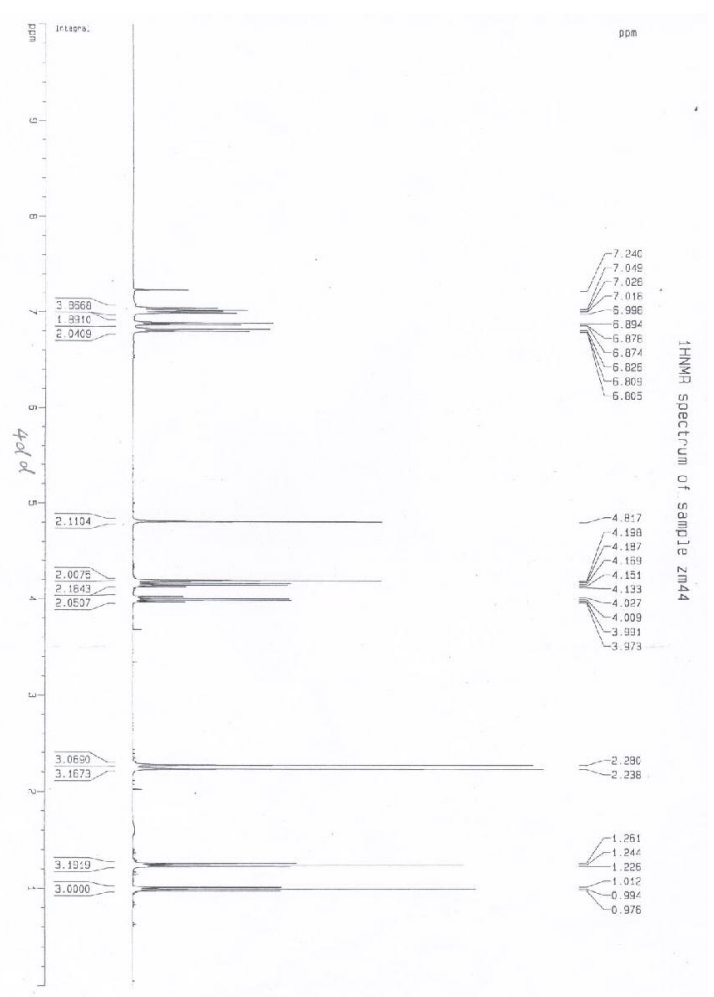

${ }^{1} \mathrm{H}$ NMR Spectrum of $4 \mathrm{ee}$ 


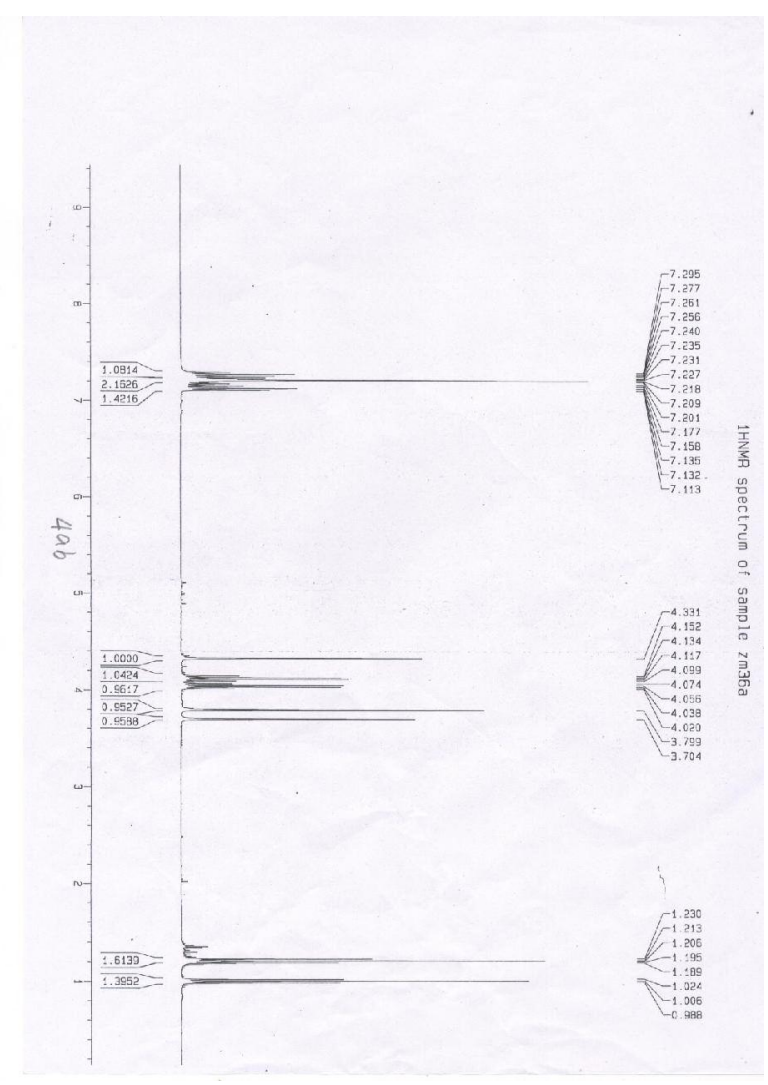

${ }^{1}$ H NMR Spectrum of $4 a b$

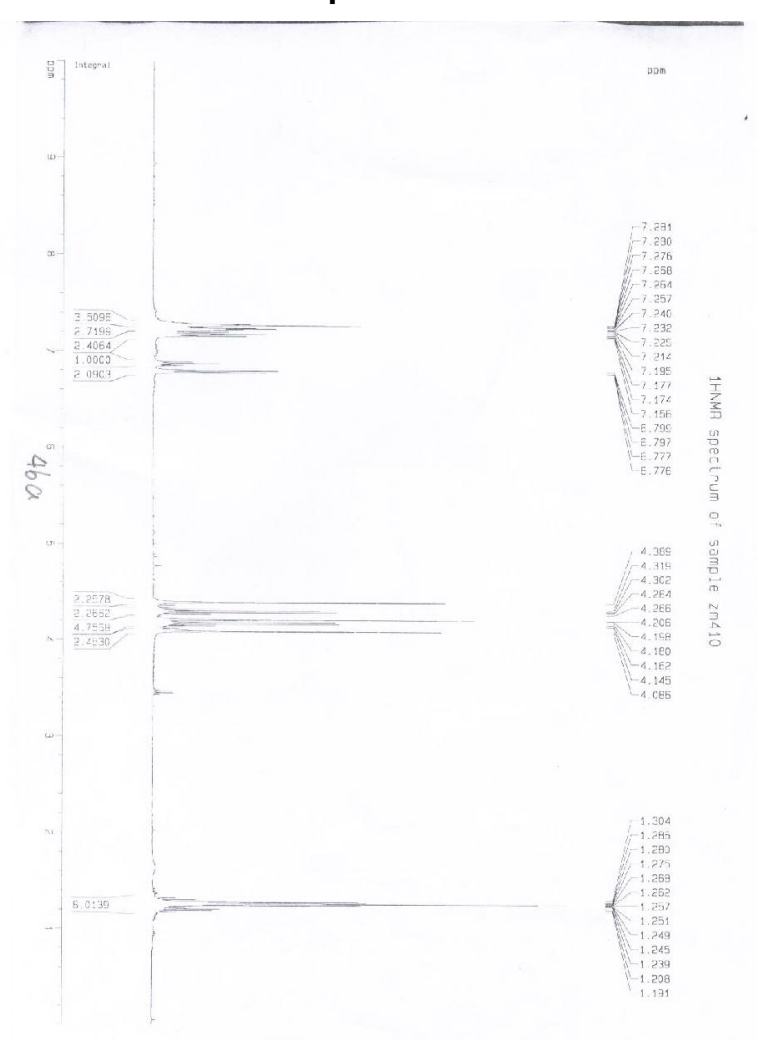

${ }^{1} \mathrm{H}$ NMR Spectrum of $4 \mathrm{ba}$ 


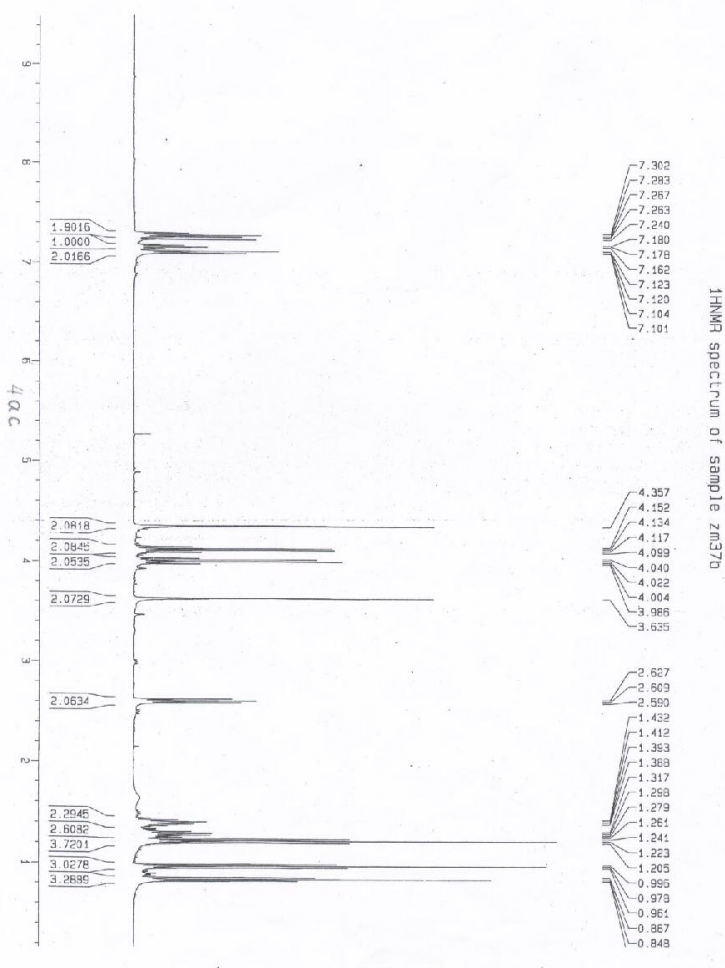

${ }^{1} \mathrm{H}$ NMR Spectrum of $4 \mathrm{ac}$ 